\title{
Microbiota bacteriana intestinal en pacientes mexicanos con inmunodeficiencia común variable
}

\author{
Ana Paola Franco-Esquivias, ${ }^{1}$ Cristina García-De la Peña, ${ }^{2 \star}$ Carlos Torres-Lozano, ${ }^{1}$ \\ Felipe Vaca-Paniagua, ${ }^{3}$ Clara Díaz-Velásquez, ${ }^{3}$ Margarita Ortega-Cisneros ${ }^{1}$ y Antonio Quintero-Ramos ${ }^{4}$ \\ ${ }^{1}$ Instituto Mexicano del Seguro Social, Centro Médico Nacional de Occidente, Hospital de Especialidades, Departamento de Alergia e Inmunología \\ Clínica, Guadalajara, Jalisco; '2Universidad Juárez del Estado de Durango, Facultad de Ciencias Biológicas, Laboratorio de Medicina de la \\ Conservación, Gómez Palacio, Durango; ${ }^{3}$ Universidad Nacional Autónoma de México, Facultad de Estudios Superiores Iztacala, Laboratorio \\ Nacional en Salud: Diagnóstico Molecular y Efecto Ambiental en Enfermedades Crónico-Degenerativas, Tlalnepantla, Estado de México; ${ }^{4}$ Universidad \\ de Guadalajara, Centro Universitario de Ciencias de la Salud, Departamento de Fisiología, Guadalajara, Jalisco, México
}

\section{Resumen}

Introducción: La inmunodeficiencia común variable (IDCV) es la principal inmunodeficiencia primaria sintomática y cursa con alteraciones inmunes complejas. La microbiota intestinal interactúa estrechamente con el sistema inmune y la disbiosis intestinal está relacionada con múltiples patologías. Objetivo: Describir por primera vez la composición de la microbiota intestinal en pacientes mexicanos con inmunodeficiencia común variable. Método: Se recolectaron muestras fecales de cinco pacientes con inmunodeficiencia común variable y se llevó a cabo secuenciación masiva de la región V3-V4 del gen 16S rRNA mediante tecnología Illumina. Resultados: Se observó abundancia bacteriana relativa a todos los niveles taxonómicos. Firmicutes, Actinobacteria y Verrucomicrobia fueron los filos predominantes. La clase Clostridia y el orden Clostridiales fueron los principales en su respectivo taxón; predominó la familia Ruminococcaceae. Se reportaron 166 géneros, el más abundante fue Faecalibacterium. Se identificaron cinco especies, pero solo Bifidobacterium longum estuvo presente en todos los pacientes. Conclusiones: A diferencia de la microbiota intestinal de sujetos sanos en quienes predominan Firmicutes y Bacteroidetes, en los pacientes con inmunodeficiencia común variable considerados en este estudio fueron abundantes Firmicutes, Actinobacterias y Verrucomicrobia. La baja abundancia de bacteroidetes y alta de firmicutes podrían significar disbiosis intestinal.

PALABRAS CLAVE: Microbiota intestinal. Inmunodeficiencia común variable. $16 S$ rRNA. Firmicutes. Disbiosis.

\begin{abstract}
Introduction: Common variable immunodeficiency (CVID) is the main symptomatic primary immunodeficiency and is associated with complex immune disorders. Gut microbiota interacts closely with the immune system, and intestinal dysbiosis is related to multiple diseases. Objective: To describe for the first time the composition of gut microbiota in Mexican patients with CVID. Methods: Fecal samples from five patients with CVID were collected and massive sequencing of the V3-V4 region of 16S rRNA gene was carried out using illumina technology. Results: Bacterial relative abundance was observed at all taxonomic levels. Firmicutes, Actinobacteria and Verrucomicrobia were the predominant phyla. The Clostridia class and the Clostridial order were the most common in their respective taxon; the Ruminococcaceae family predominated. A total of 166 genera were reported, with the most abundant being Faecalibacterium. Five species were identified, but only Bifidobacterium longum was present in all patients. Conclusions: Unlike healthy subjects' gut microbiota, where Firmicutes and Bacteroidetes predominate, the microbiota of the patients with CVID considered in this study was abundant in Firmicutes, Actinobacteria and Verrucomicrobia. The low presence of Bacteroidetes and high abundance of Firmicutes might indicate the existence of intestinal dysbiosis in these patients.
\end{abstract}

KEY WORDS: Gut microbiota. Common variable immunodeficiency. $16 S$ rRNA. Firmicutes. Dysbiosis.

Correspondencia:

${ }^{*}$ Cristina García De la Peña

E-mail: cristina.garcia@ ujed.mx
Fecha de recepción: 03-12-2018

Fecha de aceptación: 17-05-2019

DOI: 10.24875/GMM.19004879
Gac Med Mex. 2019;155:481-486

Disponible en PubMed

www.gacetamedicademexico.com 


\section{Introducción}

La inmunodeficiencia común variable (IDCV) es la inmunodeficiencia primaria sintomática más frecuente en el mundo. ${ }^{1}$ El diagnóstico se basa en los criterios propuestos por las sociedades americana y europea en inmunodeficiencias: disminución de los niveles séricos de inmunoglobulina $\mathrm{G}$ ( $\mathrm{IgG}$ ) debajo de dos desviaciones estándar para la edad y disminución de otra inmunoglobulina sérica ( $\lg A$ o $\lg M)$, edad mayor a dos años, ausencia de isohemaglutininas o pobre respuesta a vacunas y exclusión de otras causas de hipogammaglobulinemia.

La principal manifestación clínica en la IDCV es la infecciosa, sin embargo, son frecuentes otras complicaciones como autoinmunidad, trastornos gastrointestinales, granulomatosis, linfoproliferación y neoplasias. ${ }^{1-3}$ La alteración inmunológica característica es la hipogammaglobulinemia con afección a los linfocitos $B$, sin embargo, el compromiso incluye otras células como linfocitos T CD4+, linfocitos reguladores (LTreg CD25+FoxP3+), linfocitos T CD8, monocitos, células presentadoras de antígeno y células NK (natural killer). ${ }^{1,2,4} \mathrm{La}$ IDCV se ha relacionado con diversos factores genéticos y ambientales, sin embargo, ninguno ha podido explicar por completo las múltiples manifestaciones clínicas, gravedad y evolución de la enfermedad. ${ }^{1,2}$

La microbiota intestinal humana se ha propuesto como un microecosistema clave en el proceso de salud-enfermedad, en el que la disbiosis se asocia con obesidad, infecciones, autoinmunidad y enfermedades alérgicas. ${ }^{5-7}$ La evolución, desarrollo y diversidad de la microbiota intestinal inicia desde el nacimiento y se modifica a lo largo de la vida. ${ }^{8} \mathrm{El}$ $90 \%$ de los microorganismos que colonizan el intestino humano está representado por los filos Firmicutes, Bacteroides, Actinobacteria, Fusobacteria, Protebacteria y Verrucomicrobia. 9,10 Esta amplia diversidad bacteriana tiene funciones importantes en el desarrollo del sistema inmune innato y adaptativo en un proceso de retroalimentación bidireccional., ${ }^{711,12} \mathrm{~A}$ pesar de ello, el estudio de la microbiota intestinal en inmunodeficiencias primarias es limitado. Considerando la cantidad de actividades de la microbiota en la regulación inmune y las diversas alteraciones inmunológicas de la IDCV, surge la necesidad de profundizar en el conocimiento de la microbiología intestinal en pacientes con esta condición médica. Por ello, se presenta un primer análisis de la microbiota intestinal en pacientes mexicanos con IDCV, en el que se describe la estructura de la comunidad microbiana intestinal asociada con esta patología.

\section{Método}

Durante 2016 se recolectaron muestras fecales de cinco pacientes mayores de 18 años diagnosticados con IDCV pertenecientes al Departamento de Inmunología Clínica y Alergia de Centro Médico Nacional de Occidente, Instituto Mexicano del Seguro Social en Guadalajara, Jalisco, México. El protocolo fue autorizado por el comité de ética de esa institución.

Se recolectó una muestra fecal por paciente utilizando frasco estéril; se colocaron $0.25 \mathrm{~g}$ de cada muestra en tubos para lisis BashingBead ${ }^{\mathrm{TM}}$ con $750 \mu \mathrm{L}$ de buffer lisante/estabilizador Xpedition $^{\mathrm{TM}}$ Zymo Research ${ }^{\mathrm{TM}}$. Cada tubo se colocó en un disruptor celular (TerraLyzer ${ }^{\mathrm{TM}}$ ) durante 30 segundos para la conservación del ADN.

El ADN de las muestras se extrajo utilizando el kit comercial Soil/Fecal DNA MiniPrep de Zymo Research $^{\mathrm{TM}}$. EI ADN extraído se corrió en gel de agarosa a $1.2 \%$ a $80 \mathrm{~V}$ durante 45 minutos, en cámara de electroforesis BIO-RAD para visualizar la presencia de ADN de alto peso molecular. La visualización se llevó a cabo en un fotodocumentador GeIMaxTM (UVP®). La cantidad de ADN por muestra se midió en un fluorómetro marca Qubit ${ }^{\circledR}$. Se llevó a cabo la amplificación de las regiones V3 y V4 del gen 16S rRNA, empleando los primers

S-D-Bact-0341-b-S-17, 5'-CCTACGGGNGGCWGCAG-3' y S-D-Bact-0785-a-A-21, 5'-GACTACHVGGGTATCTAATCC-3', que producen un amplicón de aproximadamente 460 pb. ${ }^{13}$ Estas secuencias se sintetizaron con los adaptadores overhang del protocolo de Illumina (2017a), que producen un amplicón de aproximadamente $550 \mathrm{pb}$.

Se utilizó el protocolo de PCR Illumina con $12.5 \mu \mathrm{L}$ de MyTaq $^{\mathrm{TM}}$ Ready Mix $1 \mathrm{X}$ (Bioline $\left.{ }^{\circledR}\right), 1 \mu \mathrm{L}$ de cada primer $(10 \mu \mathrm{M}), 5 \mu \mathrm{L}$ de ADN (50 ng totales) y $5.5 \mu \mathrm{L}$ de $\mathrm{H}_{2} \mathrm{O}$ grado molecular. Se utilizó el siguiente ciclo: $95{ }^{\circ} \mathrm{C}$ por tres minutos; 25 ciclos de $95{ }^{\circ} \mathrm{C}$ por 30 segundos, $55^{\circ} \mathrm{C}$ por 30 segundos, $72{ }^{\circ} \mathrm{C}$ por 30 segundos; $72{ }^{\circ} \mathrm{C}$ por cinco minutos en un termociclador Labnet Multigene $\mathrm{T}^{\mathrm{TM}}$ Gradient PCR. Se colocó $1 \mu \mathrm{L}$ de los productos de PCR en un chip de Bioanalyzer DNA 1000 para verificar el tamaño del amplicón (aproximadamente de $550 \mathrm{pb}$ ). Se realizó la purificación de los amplicones con perlas Agentcourt ${ }^{\circledR}$ AMPure $^{\circledR}$ XP 
Tabla 1. Información de las secuencias bacterianas utilizadas en el presente estudio

\begin{tabular}{|l|l|l|l|l|l|l|l|}
\hline Paciente & ST & SE & SD & SC & SB & SSBS & OTU \\
\hline P1 & 199924 & 188675 & 11249 & 184796 & 178145 & 120665 & 6442 \\
\hline P2 & 228803 & 189333 & 39470 & 165517 & 106264 & 106264 & 5401 \\
\hline P3 & 190836 & 143260 & 47576 & 132649 & 127663 & 84229 & 5.087 \\
\hline P4 & 233012 & 177792 & 55220 & 175753 & 172519 & 131572 & 4831 \\
\hline P5 & 234330 & 154823 & 79507 & 151499 & 147932 & 108594 & 6636 \\
\hline Media & 217381 & 170777 & 46604 & 162137 & 157355 & 110265 & 5679 \\
\hline
\end{tabular}

ST = número total de secuencias, $\mathrm{SE}=$ número de secuencias ensambladas, SD = número de secuencias desechadas, SC = secuencias de calidad después de la eliminación de quimeras, $\mathrm{SB}=$ secuencias bacterianas después de la asignación taxonómica, SBSS = secuencias bacterianas después de la eliminación de los singletons, OTU = unidades taxonómicas operacionales.

a $0.8 \%$. Posteriormente, los amplicones se etiquetaron con Nextera XT Index Kit ${ }^{\mathrm{TM}}$ para la creación de las bibliotecas, empleando $25 \mu \mathrm{L}$ de $\mathrm{MyTaq}^{\mathrm{TM}}$ Ready Mix 1X (Bioline ${ }^{\circledR}$ ), $5 \mu \mathrm{L}$ de cada primer (N7xx y S5xx), $5 \mu \mathrm{L}$ de ADN y $10 \mu \mathrm{L}$ de $\mathrm{H}_{2} \mathrm{O}$ grado molecular. Se utilizó el siguiente ciclo: $95^{\circ} \mathrm{C}$ por tres minutos; 10 ciclos de $95^{\circ} \mathrm{C}$ por 30 segundos, $55^{\circ} \mathrm{C}$ por 30 segundos, $72{ }^{\circ} \mathrm{C}$ por 30 segundos, $72^{\circ} \mathrm{C}$ por cinco minutos. Se realizó la purificación de las bibliotecas con perlas Agencourt ${ }^{\circledR}$ AMPure ${ }^{\circledR}$ XP a $1.2 \%$. Se colocó $1 \mu \mathrm{L}$ de la biblioteca final de algunos productos de PCR seleccionados al azar en un chip de Bioanalyzer DNA 1000 para verificar el tamaño del amplicón, esperando un tamaño de aproximadamente 630 pb. Finalmente, se realizó cuantificación, normalización (equimolaridad), agrupación de las bibliotecas y secuenciación masiva de siguiente generación (MiSeq Illumina ${ }^{\circledR}$ de $2 \times 250$ lecturas de final pareado), conforme el protocolo para metagenómica $16 \mathrm{~S}$ de Illumina.

El análisis de las secuencias se realizó en Oracle VM VirtualBox 5.1.14 en la plataforma MGLinux mediante el software bioinformático Quantitative Insights Into Microbial Ecology, versión 1.9.0.14 El proceso se inició ensamblando las secuencias forward y reverse de las muestras, utilizando el programa $\mathrm{PEAR}^{15} \mathrm{con}$ un traslape de $50 \mathrm{pb}$, una longitud mínima por lectura de $430 \mathrm{pb}$ y máxima de $470 \mathrm{pb}$, un criterio de calidad Q30 (una base errónea por cada 1000 bases) y un valor de $p<0.0001$. Enseguida los archivos fueron convertidos a formato FASTA y se llevó a cabo la eliminación de secuencias quiméricas de las muestras con USEARCH. ${ }^{16}$ La selección de OTU (unidades taxonómicas operacionales) se realizó con el método UCLUST ${ }^{16}$ a $97 \%$ de similitud; se obtuvo una secuencia representativa para cada OTU y se asignó la taxonomía tomando como referencia la bases de datos EzBioCloud..$^{17}$ Se construyó la tabla de OTU en formato BIOM (Biological Observation Matrix), ${ }^{18}$ se separaron los dominios y se filtraron los singletons (OTU que solo tuvieron una observación)..$^{19} \mathrm{Se}$ obtuvieron las tablas de abundancia absoluta de OTU y se graficó el número de secuencias por el número de taxones a nivel género, para confirmar una profundidad de cobertura adecuada (curvas de tendencia asíntota); esta gráfica fue realizada en PAST versión $3.15 .{ }^{20}$ El número de secuencias alcanzado simultáneamente por todas las muestras fue 80000 y se llevó a cabo un proceso de rarefacción aleatoria simple,$^{21}$ tomando este valor como el número de secuencias a generarse. De esta forma se obtuvo un archivo BIOM estandarizado para todas las muestras. Se obtuvo la abundancia relativa en los niveles taxonómicos de filo, clase, orden, familia y género; los filos y los géneros fueron graficados en Excel.

\section{Resultados}

La media del total de secuencias obtenidas para todos los pacientes antes del ensamblaje fue 217381 , con una media de secuencias ensambladas de 170 777; la media de secuencias de calidad después de la eliminación de quimeras fue 162 137. Tras la asignación taxonómica se obtuvo una media de secuencias bacterianas de 157 355, que después de eliminar los singletons fue de 110 265. La media de OTU fue de 5679 (Tabla 1). La profundidad de cobertura en cuanto al número de taxones bacterianos por paciente fue adecuada, ya que se logró la tendencia a la asíntota en cada curva (Figura 1).

Se registraron 10 filos, de los cuales Firmicutes fue el más abundante $(\bar{x}=89 \%)$; Actinobacteria $(\bar{x}=6 \%)$ y Verrucomicrobia $(\bar{x}=3 \%)$ fueron los siguientes en dominancia (Figura 2). Se determinaron 15 clases, Clostridia fue la más abundante $(\bar{x}=89 \%)$, seguida 


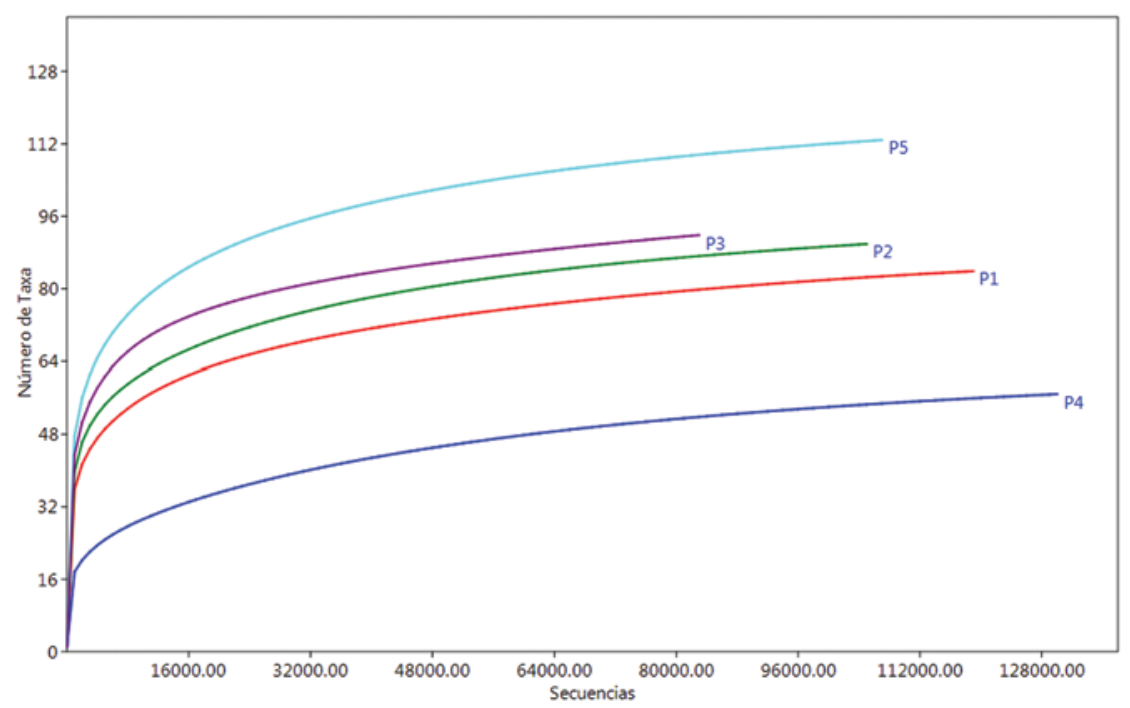

Figura 1. Profundidad de cobertura de secuencias bacterianas fecales de pacientes con inmunodeficiencia común variable.

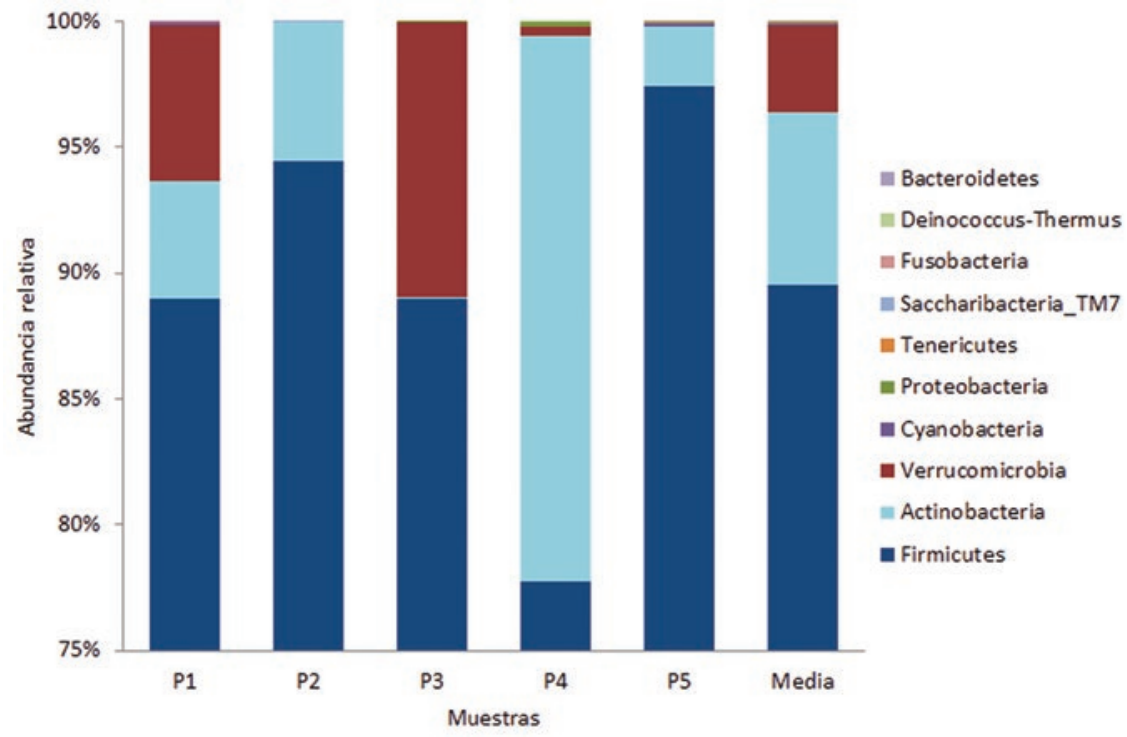

Figura 2. Abundancia relativa de los filos de bacterias fecales de pacientes con inmunodeficiencia común variable; la última barra muestra la abundancia promedio.

por una clase no identificada del filo Actinobacteria $(\bar{x}=6 \%)$ y por la clase Verrucomicrobia $(\bar{x}=3 \%)$.

Se obtuvieron 21 órdenes, Clostridiales fue el más abundante $(\bar{x}=89 \%)$, seguido por Bifidobacteriales $(\bar{x}=6 \%)$ y Verrucomicrobiales $(\bar{x}=6 \%)$. De las 34 familias registradas predominaron Ruminococcaceae $(\bar{x}=48 \%)$, Lachnospiraceae $(\bar{x}=39 \%)$, Bifidobacteriaceae $(\bar{x}=6 \%)$ y Akkermansiaceae $(\bar{x}=3 \%)$. Se reportaron 166 géneros, entre los cuales los más abundantes fueron Faecalibacterium ( $\bar{x}=23 \%)$, Blautia $(\bar{x}=7 \%)$, Ruminococcus g2 $(\bar{x}=6.3 \%) y$ Bifidobacterium ( $\bar{x}=6 \%$ ) (Figura 3$)$. Las especies identificadas fueron Bifidobacterium longum (única especie presente en $100 \%$ de los individuos),
Bifidobacterium thermacidophilum (20\% de los pacientes), Propionibacterium acnes (20\%), Eubacterium hallii (20\%) y Fusobacterium nucleatum (40\%).

\section{Discusión}

Este primer acercamiento en el estudio de la microbiota intestinal en pacientes mexicanos con IDCV difiere de lo reportado en sujetos sanos, en quienes se ha reportado que la microbiota intestinal se compone principalmente por Firmicutes y Bacteroidetes y en menor abundancia por Actinobacterias, Fusobacterias, Proteobacterias, Verrucomicrobia y Cyanobacterias. ${ }^{5,9,10,22,23}$ 


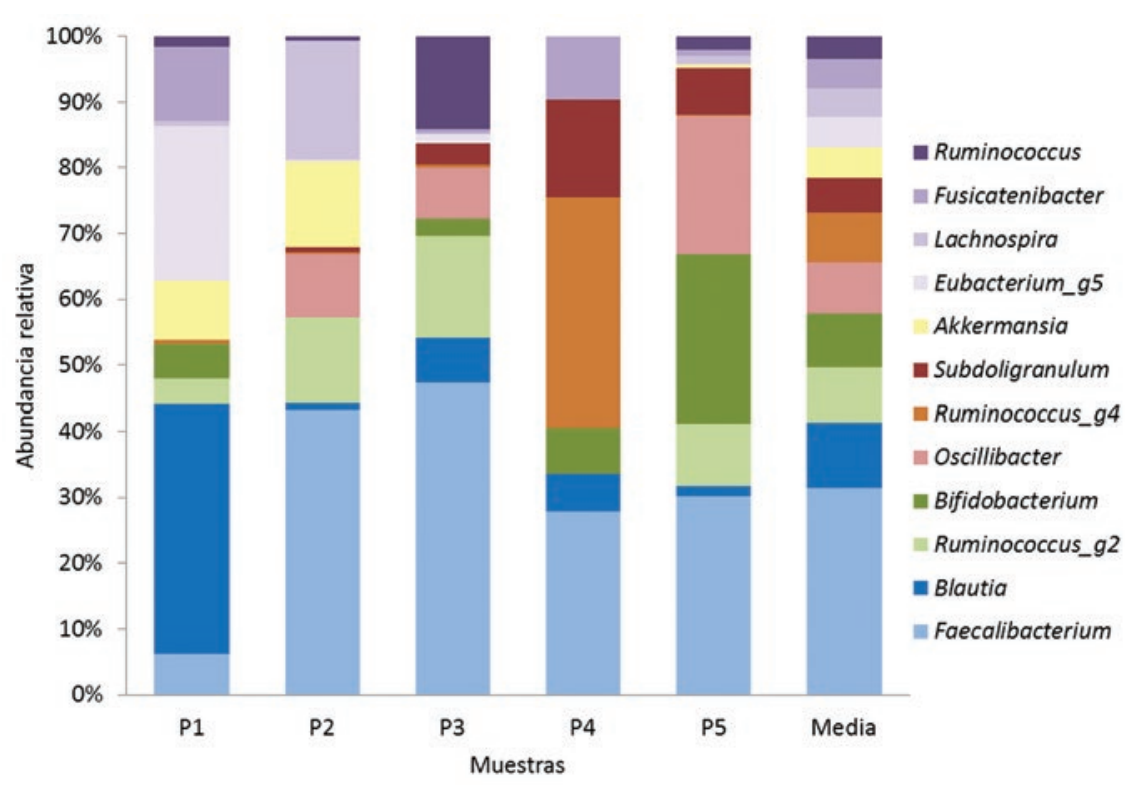

Figura 3. Abundancia relativa de los principales géneros de bacterias fecales de pacientes con inmunodeficiencia común variable; la última barra muestra la abundancia promedio.

Firmicutes, el filo más abundante en la microbiota intestinal del ser humano, ${ }^{9,23}$ abarcó casi $90 \%$ de la abundancia relativa total en el presente estudio. Derivado de este filo se identificó la clase Clostridia, las familias Ruminococcaceae y Lachnospiraceae, ocho de los principales géneros bacterianos (Faecalibacterium, Blautia, Ruminococcus, Oscillibacter, Subdoligranulum, Eubacterium, Lachnospira y Fusicatenibacter) y la especie Eubacterium hallii.

Las clases Clostridium IV y XIVa cumplen funciones inmunológicas importantes; su principal actividad se basa en la producción de ácidos grasos de cadena corta a partir de la fermentación de fibra de la dieta. ${ }^{24,25}$ Estos ácidos grasos de cadena corta se unen a los receptores para ácidos grasos libres acoplados a proteína G: GPR43/FFAR2, GPRA1/FFAR3 y GPR109A, presentes en diversas células inmunes; ${ }^{26}$ su unión estimula la diferenciación de linfocitos $T$ naive a LTreg CD25+FOXP3+ productoras de interleucina-10 y factor de crecimiento transformante beta. ${ }^{24,26}$ Estas citocinas favorecen la diferenciación de linfocitos $B$ hacia subtipos productores de $\lg A . .^{25,27-29}$

El filo Actinobacteria fue el segundo en abundancia; de él parte la clase Actinobacteria, el orden Bifidobacteriales y el género Bifidobacterium. De este filo se identificaron las especies Bifidobacterium Longum, Bifidobacterium Thermacidophilum y Propionibacterium Acnes. Bifidobacterium Longum, que se encontró en $100 \%$ de los individuos con IDCV, tiene actividades probióticas al fermentar oligosacáridos, metabolizar carbohidratos complejos y participar en la biosíntesis de polisacáridos capsulares que interactúan con los receptores tipo Toll-2 (TLR-2) en el inicio de la respuesta inmune innata. ${ }^{29,30}$ Propinobacterium acnes fermenta la lactosa en ácido propiónico, que tiene efecto antimicrobiano. ${ }^{30-32}$ Bifidobacterium thermacidophilum no ha sido reportado previamente como parte de la microbiota intestinal humana, sin embargo, este resultado debe tomarse con cautela al haberse registrado solo en un paciente.

El filo Verrucomicrobia fue el tercero en abundancia; de él se deriva la familia Akkermansiaceae y el género Akkermansia. En la mayoría de los estudios realizados en sujetos sanos (exceptuando algunos países de Latinoamérica ${ }^{33}$ ), Verrucomicrobia no se presenta con abundancia elevada, sin embargo, fue uno de los más abundantes en los individuos con IDCV. Su principal función inmunológica es mantener la integridad de la capa de moco intestinal y producir péptidos antimicrobianos. ${ }^{33,34}$

Resalta la baja abundancia del filo Bacteroidetes $(\bar{x}=0.00001)$ en los pacientes estudiados. Estos bacilos gramnegativos son importantes en el metabolismo de polisacáridos complejos que arriban intactos al colon. ${ }^{35}$

El cociente Firmicutes: Bacteroidetes se ha propuesto como indicador de disbiosis. ${ }^{36-38}$ Es evidente que la diferencia, y por lo tanto el cociente entre Firmicutes: Bacteroidetes en los pacientes con IDCV del presente estudio son extremadamente altos, hallazgo que lleva a sugerir disbiosis intestinal. 
En conclusión, se reporta por primera vez la composición de la microbiota intestinal en cinco pacientes mexicanos con IDCV. Aunque la mayoría de las bacterias identificadas tienen interacción positiva con el sistema inmune, la elevada abundancia de Firmicutes y la baja abundancia de Bacteroidetes sugieren disbiosis intestinal en estos pacientes. Aunque el tamaño de la muestra es reducido, se espera que los hallazgos reportados sean el primer paso para profundizar sobre el estudio de la microbiota en su relación con el sistema inmune. Se considera que el desarrollo de un estudio multicéntrico adicional sería de utilidad para reforzar y profundizar los hallazgos descritos.

\section{Agradecimientos}

A los pacientes que participaron en este estudio al proporcionar las muestras. Al personal del Laboratorio de Medicina de la Conservación, Facultad de Ciencias Biológicas, Universidad Juárez del Estado de Durango, Gómez Palacio, Durango, por su apoyo en la recepción, preparación y procesamiento de muestras. Al Laboratorio Nacional en Salud: Diagnóstico Molecular y Efecto Ambiental en Enfermedades Crónico-Degenerativas, Facultad de Estudios Superiores Iztacala, Universidad Nacional Autónoma de México, Tlalnepantla, Estado de México, por su apoyo tecnológico y científico en el procesamiento de las muestras.

\section{Bibliografía}

1. Bonilla FA, Barlan I, Chapel H, Costa-Carvalho BT, Cunningham-Rundles C, De la Morena MT, et al. International Consensus Document (ICON): common variable immunodeficiency disorders. J Allergy Clin Immunol Pract. 2016;4:38-59.

2. Salzer U, Warnatz K, Hartmut-Peter H. Common variable immunodeficiency. An update. Arthritis Res Ther. 2012;14:1-11.

3. Berbers R, Nierkens S, Van Laar JM Van, Bogaert D, Leavis HL. Microbial dysbiosis in common variable immune deficiencies : evidence, causes, and consequences. Trends Immunol. 2016;38:206-216.

4. Perreau M, Vigano S, Bellanger F, Pellaton C, Buss G, Comte D, et al. Exhaustion of bacteria-specific CD4 T cells and microbial translocation in common variable immunodeficiency disorders. J Exp Med. 2014;211:2033-2045.

5. Jandhyala SM, Talukdar R, Subramanyam C, Vuyyuru H, Sasikala M. Role of the normal gut microbiota. World J Gastroenterol. 2015;21:8787-8803.

6. Qin J, Li R, Raes J, Arumugam M, Burgdorf KS, Manichanh C, et al. A human gut microbial gene catalogue established by metagenomic sequencing. Nature. 2010;464:59-65

7. Cénit MC, Matzaraki V, Tigchelaar EF, Zhernakova A. Rapidly expanding knowledge on the role of the gut microbiome in health and disease. Biochim Biophys Acta. 2014;1842:1981-1992.

8. Belkaid $Y$, Hand TM. Role of the microbiota in immunity and inflamation. Cell. 2014;157:121-141.

9. lizumi T, Battaglia T, Ruiz V, Pérez-Pérez GI. Gut microbiome and antibiotics. Arch Med Res. 2017:48:727-734.

10. Arumugam M, Raes J, Pelletier E, Le Paslier D, Batto J, Yamada T, et al. Enterotypes of the human gut microbiome. Nature. 2011;473:174-180.
11. Shulzhenko N, Morgun A, Hsiao W, Battle M, McCoy D, Fraser-Liggett C, et al. Crosstalk between B lymphocytes, microbiota and the intestinal ephitelium governs immunity versus metabolism in the gut. Nat Med. 2014;17:1585-1593.

12. Jørgensen SF, Trøseid M, Kummen M, Anmarkrud JA, Michelsen AE, Osnes LT, et al. Altered gut microbiota profile in common variable immunodeficiency associates with levels of lipopolysaccharide and markers of systemic immune activation. Mucosal Immunol. 2016;9:1455-1465.

13. Klindworth A, Pruesse E, Schweer T, Horn M, Schweer T. Evaluation of general 16S ribosomal RNA gene PCR primers for classical and next-generation sequencing-based diversity studies. Nucleic Acids Res. 2013:41:e1.

14. Caporaso JG, Kuczynski J, Stombaugh J, Bittinger K, Bushman FD, Costello EK, et al. QIIME allows analysis of high-throughput community sequencing data. Nat Methods. 2010;7:335-336.

15. Stamatakis A, Zhang J, Kobert K, Flouri T. Genome analysis PEAR : a fast and accurate Illumina Paired-End reAd mergeR. Bioinformatics. 2014;30:614-620.

16. Edgar RC. Search and clustering orders of magnitude faster than BLAST. Bioinformatics. 2010;26:2460-2461.

17. Yoon S, Ha S, Kwon S, Lim J, Kim Y, Seo H, et al. Introducing EzBioCloud: a taxonomically united database of 16S rRNA gene sequences and whole-genome assemblies. Int J Sysy Evol Microbiol. 2017;1613-1617.

18. Mcdonald D, Clemente JC, Kuczynski J, Rideout JR, Stombaugh J, Wendel $D$, et al. The Biological Observation Matrix (BIOM) format or: how I learned to stop worrying and love the ome-ome. Gigascience. 2012;1(1): 7.

19. Navas-Molina J, Peralta-Sánchez J, González A, McMurdie PJ, Vázquez-Baeza $Y, X u Z Z$, et al. Advancing our understanding of the human microbiome using QIIME. Methods Enzymol. 2015;531:371-444.

20. Hammer $\varnothing$, Harper DAT, Ryan PD. PAST: Paleontological Statistics Software Package For Education and Data Analysis. Palaentologia Electron. 2001;4:1-9.

21. Weiss S, Xu ZZ, Peddada S, Amir A, Bittinger K, Gonzalez A, et al. Normalization and microbial differential abundance strategies depend upon data characteristics. Microbiome; 2017;5:27.

22. Ottman N, Smidt H, De Vos WM De, Belzer C. The function of our microbiota : who is out there and what do they do? Front Cell Infect Microbiol. 2012;2:1-11.

23. Ghazalpour A, Cespedes I, Bennett BJ, Allayee H. Expanding role of gut microbiota in lipid metabolism. Curr Opin Lipidol. 2016;27:141-147.

24. Lopetuso LR, Scaldaferri F, Petito V, Gasbarrini A. Commensal clostridia: leading players in the maintenance of gut homeostasis. Gut Pathog. 2013;5:1.

25. Engels $\mathrm{C}$, Ruscheweyh HJ, Beerenwinkel N, Lacroix C, Schwab C. The common gut microbe Eubacterium hallii also contributes to intestinal propionate formation. Front Microbiol. 2016;7:1-12.

26. Shukla SD, Budden KF, Neal R, Hansbro PM. Microbiome effects on immunity, health and disease in the lung. Clin Transl Immunology. 2017:6:e133.

27. Kawamoto S, Maruya M, Kato LM, Suda W, Atarashi K, Doi Y, et al. Foxp3 + T cells regulate immunoglobulin a selection and facilitate diversification of bacterial species responsible for immune homeostasis. Immunity. 2014;41:1-14.

28. Ray A, Dittel BN. Interrelatedness between dysbiosis in the gut microbiota due to immunodeficiency and disease penetrance of colitis. Immunology. 2015;146:359-368

29. Wu H, Wu E. Health technology slide shows. Web feature gets down to basics. Health Devices. 2003;32:126-127.

30. Ventura M, Canchaya C, Tauch A, Chandra G, Fitzgerald GF, Chater KF et al. Genomics of actinobacteria: tracing the evolutionary history of an ancient phylum. Microbiol Mol Biol Rev. 2007;71:495-548.

31. Salem I, Ramser A, Isham N, Ghannoum MA. The gut microbiome as a major regulator of the gut-skin axis. Front Microbiol. 2018;9:1-14.

32. Achermann Y, Goldstein EJC, Coenye T, Shirtliffa ME. Propionibacterium acnes: from commensal to opportunistic biofilm-associated implant pathogen. Clin Microbiol Rev. 2014;27:419-440.

33. Fujio-Vejar S, Vasquez Y, Morales P, Magne F, Vera-Wolf P, Ugalde JA, et al. The gut microbiota of healthy Chilean subjects reveals a high abundance of the phylum verrucomicrobia. Front Microbiol. 2017:8:1-11.

34. Dubourg G, Lagier JC, Armougom F, Robert C, Audoly G, Papazian L, et al. High-level colonisation of the human gut by Verrucomicrobia following broad-spectrum antibiotic treatment. Int J Antimicrob Agents. 2013;41:149-155.

35. Wexler HM. Bacteroides: the good, the bad, and the nitty-gritty. Clin Microbiol Rev. 2007;20:593-621.

36. Tamboli CP, Neut C, Desreumaux P, Colombel JF. Dysbiosis in inflammatory bowel disease. Gut. 2004:53:1-4.

37. Collins SM. A role for the gut microbiota in IBS. Nat Rev Gastroenterol Hepatol. 2014;11:497-505.

38. Sampson TR, Debelius JW, Thron T, Janssen S, Shastri GG, Ilhan ZE, et al. Gut microbiota regulate motor deficits and neuroinflammation in a model of Parkinson's disease. Cell. 2016;167:1469-1480. 